\title{
Temporal Volume Change of Cavernous Sinus Cavernous Hemangiomas after Gamma Knife Surgery
}

\author{
Jin Mo Cho ${ }^{1}$, Kyoung Su Sung², In-Ho Jung ${ }^{3}$, Won Seok Chang, ${ }^{3,4}$ Hyun Ho Jung ${ }^{3,4}$, and Jong Hee Chang ${ }^{3,4,5}$ \\ ${ }^{1}$ Department of Neurosurgery, International St Mary's Hospital, Catholic Kwandong University, Incheon; \\ ${ }^{2}$ Department of Neurosurgery, Dong-A University Hospital, Dong-A University College of Medicine, Busan; \\ ${ }^{3}$ Department of Neurosurgery, ${ }^{4} \mathrm{Gamma}$ Knife Center, ${ }^{5}$ Brain Tumor Center, Yonsei University Health System, Seoul, Korea.
}

Cavernous hemangiomas occur very rarely in the cavernous sinus. This study aimed to evaluate the efficacy of Gamma Knife surgery (GKS) on cavernous sinus cavernous hemangioma ( $\mathrm{CSCH}$ ) and to analyze the temporal volume change. We retrospectively reviewed the clinical data of $26 \mathrm{CSCH}$ patients who were treated with GKS between 2001 and 2017. Before GKS, 11 patients (42.3\%) had cranial neuropathies and 5 patients $(19.2 \%)$ complained of headache, whereas 10 patients (38.5\%) were initially asymptomatic. The mean pre-GKS mass volume was $9.3 \mathrm{~mL}$ (range, $0.5-31.6 \mathrm{~mL}$ ), and the margin dose ranged from 13 to $15 \mathrm{~Gy}$ according to the mass volume and the proximity to the optic pathway. All cranial neuropathy patients and half of headache patients showed clinical improvement. All 26 patients achieved mass control; remarkable responses (less than $1 / 3$ of the initial mass volume) were shown in 19 patients (73.1\%) and moderate responses (more than $1 / 3$ and less than $2 / 3$ ) in 7 patients (26.9\%). The mean final mass volume after GKS was $1.8 \mathrm{~mL}$ (range, $0-12.6 \mathrm{~mL}$ ). The mean mass volume at 6 months after GKS was $45 \%$ (range, 5-80\%) compared to the mass volume before GKS and $21 \%$ (range, 0-70\%) at 12 months. The higher radiation dose tended to induce more rapid and greater volume reduction. No treatment-related complication was observed during the follow-up period. GKS could be an effective and safe therapeutic strategy for CSCH. GKS induced very rapid volume reduction compared to other benign brain tumors.

Key Words: Cavernous sinus, cavernous hemangiomas, cranial nerve palsy, radiosurgery

Cavernous sinus cavernous hemangioma $(\mathrm{CSCH})$ is a relatively rare benign lesion of the cavernous sinus. Despite its benign nature, it can manifest symptoms that can result from progressive mass growth and compression of cranial nerves. CSCHs could be misdiagnosed as meningioma or schwannomas. ${ }^{1-5}$ Magnetic resonance image (MRI) and red blood cell (RBC) scan are commonly used for differential diagnosis of these lesions. ${ }^{6}$

Surgical removal of CSCH can cause many morbidities or

Received: March 10, 2020 Revised: September 15, 2020

Accepted: September 25, 2020

Corresponding author: Jong Hee Chang, MD, PhD, Department of Neurosurgery, Gamma Knife Center, Brain Tumor Center, Yonsei University Health System, 50-1 Yonsei-ro, Seodaemun-gu, Seoul 03722, Korea.

Tel: 82-2-2228-2162, Fax: 82-2-393-9979, E-mail: changjh@yuhs.ac

-The authors have no potential conflicts of interest to disclose.

(c) Copyright: Yonsei University College of Medicine 2020

This is an Open Access article distributed under the terms of the Creative Commons Attribution Non-Commercial License (https://creativecommons.org/licenses/ by-nc/4.0) which permits unrestricted non-commercial use, distribution, and reproduction in any medium, provided the original work is properly cited. sometimes mortality due to increased mass vascularization; the surgical mortality rate associated with $\mathrm{CSCH}$ is reported to be $38 \%$. ${ }^{3}$ With advances in neurosurgical techniques, the risk is decreasing; however, surgical removal of $\mathrm{CSCH}$ is still challenging. Since its discovery, Gamma Knife surgery (GKS) has been widely used to manage $\mathrm{CSCH}$ and avoid the surgery-related risks. The purpose of this study was to evaluate the efficacy and safety of GKS treatment of CSCH with a specific focus on their relationship with radiological results and clinical outcomes.

This study included all adult patients (age $>18$ years) who underwent stereotactic radiosurgery for a radiologically suspected CSCH between 2001 and 2017 at the Department of Neurosurgery, Severance Hospital, Yonsei University. Patients with insufficient clinical data as well as patients with insufficient follow-up time (less than 12 months) were excluded from the final analysis.

All CSCHs were diagnosed based on radiologic findings, except for one case of histopathologic diagnosis at the previous surgery. The diagnosis was made by experienced neuroradiol- 
ogists based on typical MRI findings, including 1) high signal intensities on T2-weighted images, 2) strong delayed enhancement after contrast injection, and 3) a round shape without a "dural tail" sign to exclude the possibility of meningioma. ${ }^{7,8} \mathrm{In}$ cases where the diagnosis was difficult to confirm using MRI scan, labeled red blood cell pool scintigraphy (RBC scan) was used for differential diagnosis. ${ }^{6,7}$ This study showed progressive and persistent tracer accumulation of labeled RBCs in the lesion, establishing RBC scan as an effective method for the diagnosis of $\mathrm{CSCH}^{6,7}$

Single-session GKS was provided to all of the patients using the Leksell Gamma Knife (Elekta Instrument AB, Stockholm, Sweden), Model B, C, or Perfexion. Following the frame fixation, a T1-weighted, three-dimensional, multiplanar, rapid-acquisition, gradient-echo MRI and a T2-weighted sequence were obtained before and after gadolinium enhancement. Those images were exported to a computer workstation for dose planning using the Gamma Plan software (Elekta Instrument $A B$ ). The GKS dose was determined according to the proximity to the optic pathway and the mass volume calculated during the dose planning. Multiple isocenter planning method was applied to minimize the radiation exposure to the critical neuronal structures, such as the optic nerve.

All GKS were outpatient-based, and performed with a routine clinical follow-up schedule, as follows: clinical follow-up at 4 weeks after GKS, imaging follow-up at 6 and 12 months after GKS, and then annual follow-up. Specifically, in case of sustained symptoms, imaging follow-up was performed, regardless of the scheduled timeline.

We evaluated the changes in mass volume, symptom improvement, and adverse effects induced by GKS at regular follow-up. T1-weighted gadolinium-enhanced image and T2-weighted image were used for mass volume measurement. The mass volume change was defined as the ratio of the follow-up mass volume to the initial mass volume. "Remarkable response" was defined as the follow-up mass volume less than $1 / 3$ of the initial mass volume, "moderate response" as the follow-up mass volume more than $1 / 3$ and less than $2 / 3$, and "minimal response" as the follow-up mass volume more than $2 / 3$. As for the patient's recovery, "complete recovery" was defined as the disappearance of symptoms before treatment, "partial recovery" as any improvement in clinical feature, and "no change" as no clinical deterioration or improvement observed after treatment.

We used the SPSS version 21.0 (IBM Corp., Armonk, NY, USA) for statistical analyses and the Wilcoxon paired $t$ test to analyze the volume change data. $P$-value less than 0.05 was considered statistically significant.

Among the 29 patients who were treated by GKS, three patients missed a follow-up MRI due to general clinical improvement, as they personally declared. As a result, they were excluded from this study, and a final total of $26 \mathrm{CSCH}$ patients were included in this study. Among the 26 patients, GKS was performed for 24 patients as a primary treatment, and a second- ary treatment for two patients (one residual mass after surgical resection and one recurred mass after conventional radiation therapy). The median age of the patients at the time of treatment was 54 years (range, 28-75 years), and the majority were female $(\mathrm{n}=20,76.9 \%)$.

Patient demographics and outcomes are described in Table 1. Before treatment, 11 patients (42.3\%) had cranial nerve dysfunction characterized by ptosis, diplopia, facial sense change, or vision change; and 5 patients (19.2\%) experienced headache. In contrast, the remaining 10 patients (38.5\%) were incidentally diagnosed during their workup for assessing other conditions, such as head injury. The mean clinical follow-up period was 45.7 months (range, 12.1-131.1 months), and the mean mass volume before GKS was $9.3 \mathrm{~mL}$ (range, $0.5-31.6 \mathrm{~mL}$ ). The mean marginal dose directed to the $50 \%$ isodose line was $13.7 \mathrm{~Gy}$ (range, 13-15 Gy). Clinical results showed good clinical outcomes in all patients; 10 patients had "complete recovery," 6 patients had "partial recovery," and 10 patients had "no change." Moreover, there was no recurrence or aggravation of symptoms during the follow-up period. None of the patients showed minimal response, and all 26 patients achieved mass control; remarkable responses were observed in 19 patients $(73.1 \%)$, and moderate responses in 7 patients $(26.9 \%)$. The mean mass volume at 6 months after GKS was $45 \%$ (range, 5-80\%) of the mass volume before GKS and $21 \%$ (range, $0-70 \%$ ) at 12 months. PostGKS MRI in 26 patients revealed a mean post-treatment mass volume of $1.8 \mathrm{~mL}$ (range, $0-12.6 \mathrm{~mL}$ ), which was significantly lower than the pre-treatment volumes $(p<0.05)$. Fig. 1 shows

Table 1. Patient Demographics and Outcomes

\begin{tabular}{lc}
\hline \multicolumn{1}{c}{ Variables } & Patients (n=26) \\
\hline Sex (\%) & $6(23.1)$ \\
Male & $20(76.9)$ \\
\hline Female & $54.9 \pm 13.3$ \\
Age (yr) & \\
Side (\%) & $17(65.4)$ \\
\hline Left & $9(34.6)$ \\
\hline Right & $45.7 \pm 27.7$ \\
Follow-up duration (months) & $13.7 \pm 0.6$ \\
Marginal dose (Gy) & $9.3 \pm 7.3$ \\
Mass volume before GKS (mL) & $1.8 \pm 2.9$ \\
Mass volume after GKS (mL) & $20.8 \pm 19.5$ \\
Mass volume after GKS (\%) & $19(73.1)$ \\
Radiologic results* $(\%)$ & $7(26.9)$ \\
\hline Remarkable response $(<1 / 3)$ & $0(0.0)$ \\
\hline Moderate response $(1 / 3-2 / 3)$ & $10(38.5)$ \\
\hline Minimal response $(>2 / 3)$ & $6(23.1)$ \\
Clinical results (\%) & $10(38.5)$ \\
\hline Complete recovery & \\
\hline Partial recovery &
\end{tabular}

GKS, Gamma Knife surgery.

${ }^{*}$ Final follow-up volume compared to pre-GKS volume. 
temporal volume change after GKS during follow-up.

Then, we classified the 26 patients into two groups according to their radiologic results to identify the factors that influence mass volume change after GKS; 7 patients were classified as moderate remission group and 19 patients as remarkable remission group. We checked to see whether there were any significant differences between the two groups using the chisquare or Student's t-test (Table 2). Naturally, the mass volume after GKS showed a significant difference between the two groups ( $p=0.044, p<0.001)$. The marginal dose tended to be higher in the remarkable remission group than in the moderate remission group, but it did not reach statistical significance. We expect that if more sample sizes are secured, the results would have statistical significance. Other variables did not show statistical difference.

Cavernous hemangioma in cavernous sinus is a rarely pre-

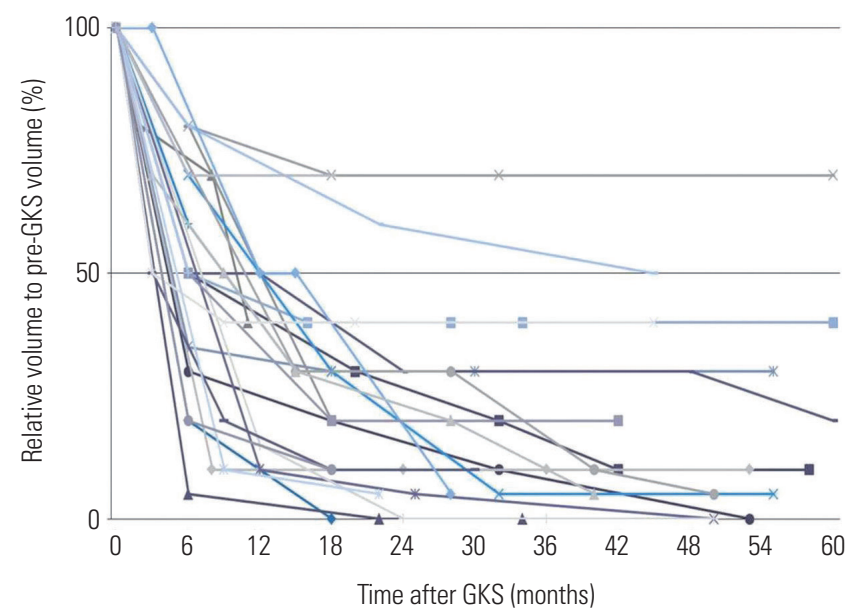

Fig. 1. Temporal volume changes after GKS in 26 patients. The graph shows rapid mass decrease (within 6-12 months after GKS). The mass volume gradually decreased over 2 years, and no volume re-expansion was observed. GKS, Gamma Knife surgery.

Table 2. Comparisons between Moderate Remission Group and Remarkable Remission Group

\begin{tabular}{|c|c|c|c|}
\hline \multirow[b]{2}{*}{ Variables } & \multicolumn{3}{|c|}{ Radiologic results } \\
\hline & $\begin{array}{c}\text { Moderate } \\
\text { remission } \\
\quad(n=7)\end{array}$ & $\begin{array}{c}\text { Remarkable } \\
\text { remission } \\
\text { (n=19) }\end{array}$ & $p$ value \\
\hline $\operatorname{Sex}(\%)$ & & & 0.904 \\
\hline Male & $1(14.3)$ & $5(26.3)$ & \\
\hline Female & $6(85.7)$ & 14 (73.7) & \\
\hline Age (yr) & $60.5 \pm 10.7$ & $52.8 \pm 13.8$ & 0.201 \\
\hline Side (\%) & & & 1.000 \\
\hline Left & $5(71.4)$ & $12(63.2)$ & \\
\hline Right & $2(28.6)$ & $7(36.8)$ & \\
\hline Marginal dose (Gy) & $13.3 \pm 0.5$ & $13.8 \pm 0.6$ & 0.069 \\
\hline Mass volume before GKS (mL) & $10.2 \pm 10.2$ & $8.9 \pm 6.2$ & 0.698 \\
\hline Mass volume after GKS (mL) (\%) & $\begin{array}{c}4.7 \pm 4.0 \\
(48.6 \pm 9.0)\end{array}$ & $\begin{array}{c}8.0 \pm 1.3 \\
(10.5 \pm 9.6)\end{array}$ & \\
\hline
\end{tabular}

GKS, Gamma Knife surgery. sented lesion, which shares similar histologic, but different clinical features with intracerebral lesions. Many well-known neurosurgeons have reported their clinical experience of surgical removal of CSCHs, and specifically, suggested a total removal rate of $40-92.3 \% .^{2,8-11}$ Even though these surgeries were performed by very experienced neurosurgeons, the reported postoperative morbidity and mortality rates were as high as $80 \%$ and $20 \%$, respectively. ${ }^{2,5,8,9,12}$ The reasons for the poor surgical outcomes were the high mass vascularization and deep location.

Before the era of GKS, radiotherapy was considered as an alternative treatment modality for avoiding the surgical risk. A number of studies have reported good clinical outcomes after radiotherapy, with a relatively high dose of radiation ( $>3 \mathrm{~Gy}$ ) in fractions considered as an effective dose for CSCH management. ${ }^{3,13-15}$ However, radiation therapy can cause complications in the central nervous system, especially after high-dose radiation.

According to Iwai, et al., ${ }^{16} \mathrm{CSCH}$ was firstly treated with GKS in 1999. The patient was surgically treated at first, and then, GKS was performed for the residual lesion, resulting in good clinical outcome. Following that case, many studies suggested that GKS could be an alternative to surgery or conventional radiotherapy. ${ }^{17-22}$

Wang, et al. ${ }^{22}$ published the meta-analysis results of GKS for $59 \mathrm{CSCH}$ patients. Their study reported a remarkable mass shrinkage (more than $50 \%$ ) in 40 patients (67.8\%), partial shrinkage (25-50\%) in 15 patients (25.4\%), and no change (less than $25 \%$ ) in 4 patients $(6.8 \%)$. They also reported that there was no significant correlation between lesion volume and mass shrinkage. However, patients with remarkable mass shrinkage were associated with higher prescription radiation dose (14 Gy vs. 13.5 Gy, $p=0.031$ ).

Our study also demonstrated remarkable mass shrinkage during relatively early follow-up. Specifically, significant shrinkage was observed in 19 patients (73.1\%), clinical symptoms were relieved within a short period after GKS for about half of the patients (53.8\%), and no complication related to GKS was noted. These results were consistent with previous studies, and the rapid clinical improvement could be a result of rapid volume reduction (Fig. 2).

The optimal radiation dose for mass control and symptom relief still remains debatable. The exposure to higher radiation doses facilitates higher rates of mass control, but it can negatively affect critical structures around the cavernous sinus, such as the optic apparatus or cranial nerves. Therefore, the possibility of this complication usually limits the exposure to high radiation doses. ${ }^{22}$ Our results showed that a higher radiation dose tended to induce earlier and greater volume reduction.

Our study had several limitations. First, CSCHs were not diagnosed according to pathologic findings, but imaging findings. Therefore, there was a small possibility of misdiagnosis, and we tried to minimize the risk of misdiagnosis by performing RBC scan in case of difficult differentiation from other pathology. 

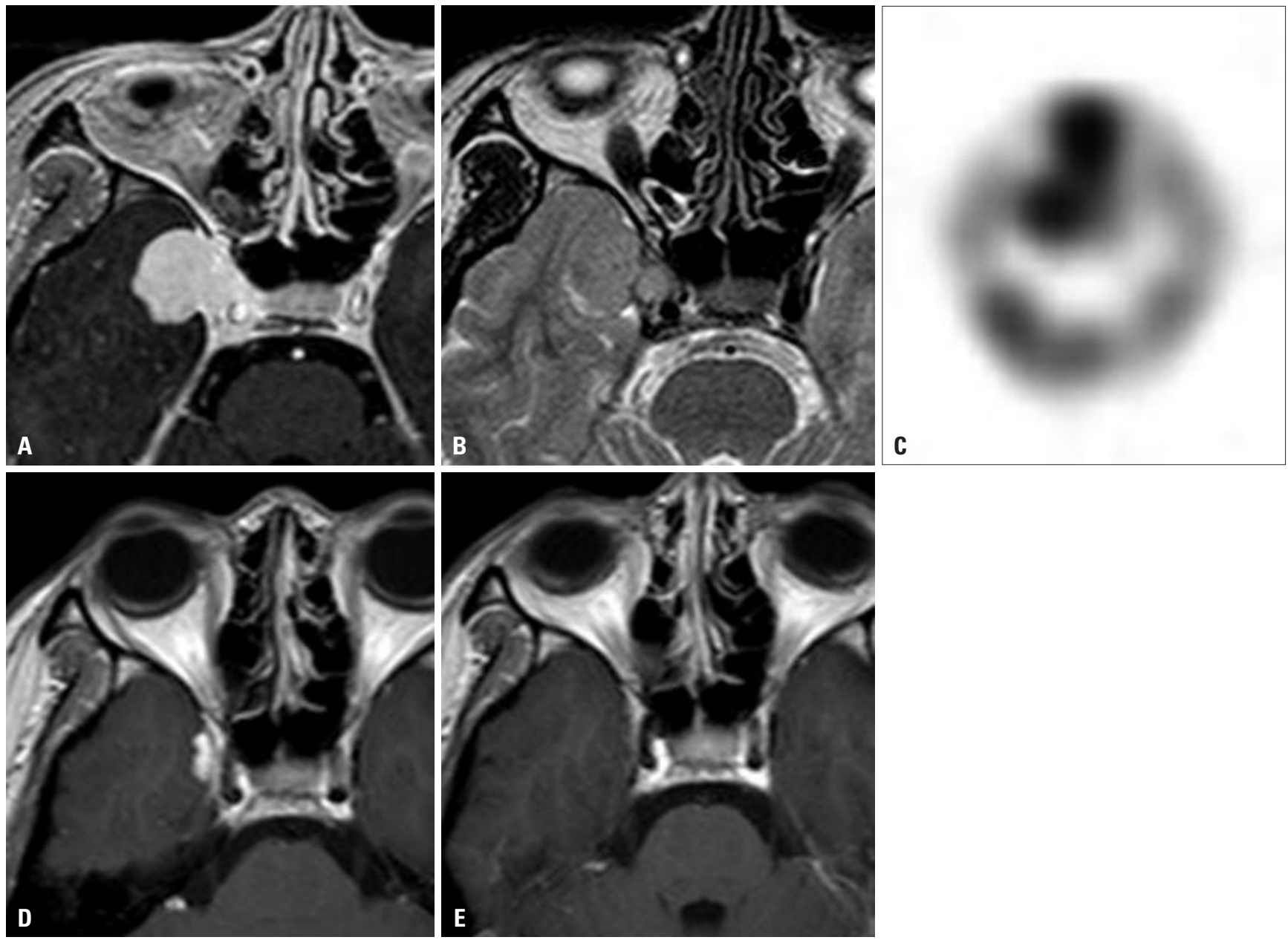

Fig. 2. A 35-year-old female patient presented with incomplete right 6th cranial nerve palsy. T1-weighted (A) and T2-weighted images (B) showed a mass at the right cavernous sinus. Red blood cell scintigraphy $(\mathrm{C})$ showed marked radioisotope uptake at the lesion, confirming the diagnosis of cavernous sinus cavernous hemangioma. The measured volume was $5.3 \mathrm{~mL}$. After GKS ( $14 \mathrm{~Gy}$ of marginal dose at the $50 \%$ isodose line), all symptoms were resolved within 1 week. The 6-month follow-up magnetic resonance image showed remarkable decrease of the lesion (D), while the lesion could be hardly seen at 35 months after GKS (E). GKS, Gamma Knife surgery.

Second, as about half of the patients in our series were incidentally diagnosed or had no cranial nerve deficit, the rationality of upfront GKS for these lesions could be controversial. Nevertheless, the minimal invasiveness and higher safety of GKS could be a rationale for treating CSCHs that are incidentally found, as well.

Although surgical resection for $\mathrm{CSCH}$ is a curative treatment, it is not always easy and safe. Due to the highly radiosensitive nature of $\mathrm{CSCH}$, GKS could be an effective and safe primary treatment modality for CSCH to prevent possible surgical complications. Further studies should be performed to define the natural history of and optimal treatment guidelines for $\mathrm{CSCH}$.

\section{AUTHOR CONTRIBUTIONS}

Conceptualization: Jin Mo Cho, Won Seok Chang, Hyun Ho Jung, and Jong Hee Chang. Data curation: Jin Mo Cho and Kyoung Su Sung. Formal analysis: Jin Mo Cho and In-Ho Jung. Funding acquisition: Jong Hee Chang. Investigation: Jin Mo Cho and In-Ho Jung. Method- ology: Jin Mo Cho and Jong Hee Chang. Project administration: Jong Hee Chang. Resources: Won Seok Chang, Hyun Ho Jung, and Jong Hee Chang. Software: Jin Mo Cho and In-Ho Jung. Supervision: Jong Hee Chang. Validation: Won Seok Chang, Hyun Ho Jung, and Jong Hee Chang. Visualization: Jin Mo Cho, In-Ho Jung, and Jong Hee Chang. Writing_original draft: Jin Mo Cho and Jong Hee Chang. Writing_review \& editing: Jin Mo Cho, In-Ho Jung, and Jong Hee Chang. Approval of final manuscript: all authors.

\section{ORCID iDs}

Jin Mo Cho https://orcid.org/0000-0002-1192-8993 Kyoung SuSung https://orcid.org/0000-0003-3289-0143 In-Ho Jung https://orcid.org/0000-0002-4135-5743 Won Seok Chang https://orcid.org/0000-0003-3145-4016 Hyun Ho Jung https://orcid.org/0000-0002-8289-564X Jong Hee Chang https://orcid.org/0000-0003-1509-9800

\section{REFERENCES}

1. Gonzalez LF, Lekovic GP, Eschbacher J, Coons S, Porter RW, Spet- 
zler RF. Are cavernous sinus hemangiomas and cavernous malformations different entities? Neurosurg Focus 2006;21:e6.

2. Zhou LF, Mao Y, Chen L. Diagnosis and surgical treatment of cavernous sinus hemangiomas: an experience of 20 cases. Surg Neurol 2003;60:31-6; discussion 36-7.

3. Linskey ME, Sekhar LN. Cavernous sinus hemangiomas: a series, a review, and an hypothesis. Neurosurgery 1992;30:101-8.

4. Li P, Ren H, Zhang S, Wang W. Clinical results of Gamma Knife surgery for cavernous sinus hemangiomas. J Neurosurg 2012;117 Suppl:89-95.

5. Dou Y, Meng Q, Yan Z, Xu J, Che S, Jiao Y, et al. Diagnosis and microsurgical treatment of cavernous sinus hemangioma. Artif Cells Blood Substit Immobil Biotechnol 2010;38:109-12.

6. Salanitri GC, Stuckey SL, Murphy M. Extracerebral cavernous hemangioma of the cavernous sinus: diagnosis with MR imaging and labeled red cell blood pool scintigraphy. AJNR Am J Neuroradiol 2004;25:280-4.

7. Polito E, Burroni L, Pichierri P, Loffredo A, Vattimo AG. Technetium tc $99 \mathrm{~m}$-labeled red blood cells in the preoperative diagnosis of cavernous hemangioma and other vascular orbital tumors. Arch Ophthalmol 2005;123:1678-83.

8. Shi J, Hang C, Pan Y, Liu C, Zhang Z. Cavernous hemangiomas in the cavernous sinus. Neurosurgery 1999;45:1308-13; discussion 1313-4.

9. Ohata K, El-Naggar A, Takami T, Morino M, El-Adawy Y, El-Sheik $\mathrm{K}$, et al. Efficacy of induced hypotension in the surgical treatment of large cavernous sinus cavernomas. J Neurosurg 1999;90:702-8.

10. Bansal S, Suri A, Singh M, Kale SS, Agarwal D, Sharma MS, et al. Cavernous sinus hemangioma: a fourteen year single institution experience. J Clin Neurosci 2014;21:968-74.

11. Goel A. The extradural approach to lesions involving the cavernous sinus. Br J Neurosurg 1997;11:134-8.

12. Suri A, Ahmad FU, Mahapatra AK. Extradural transcavernous ap- proach to cavernous sinus hemangiomas. Neurosurgery 2007;60: 483-8; discussion 488-9.

13. Shibata S, Mori K. Effect of radiation therapy on extracerebral cavernous hemangioma in the middle fossa. Report of three cases. J Neurosurg 1987;67:919-22.

14. Rigamonti D, Pappas CT, Spetzler RF, Johnson PC. Extracerebral cavernous angiomas of the middle fossa. Neurosurgery 1990;27: 306-10.

15. Maruishi M, Shima T, Okada Y, Nishida M, Yamane K, Okita S. Cavernous sinus cavernoma treated with radiation therapy--case report. Neurol Med Chir (Tokyo) 1994;34:773-7.

16. Iwai Y, Yamanaka K, Nakajima H, Yasui T. Stereotactic radiosurgery for cavernous sinus cavernous hemangioma. Neurol Med Chir (Tokyo) 1999;39:288-90.

17. Chou CW, Wu HM, Huang CI, Chung WY, Guo WY, Shih YH, et al. Gamma knife surgery for cavernous hemangiomas in the cavernous sinus. Neurosurgery 2010;67:611-6; discussion 616.

18. Yamamoto M, Kida Y, Fukuoka S, Iwai Y, Jokura H, Akabane A, et al. Gamma Knife radiosurgery for hemangiomas of the cavernous sinus: a seven-institute study in Japan. J Neurosurg 2010;112:772-9.

19. Thompson TP, Lunsford LD, Flickinger JC. Radiosurgery for hemangiomas of the cavernous sinus and orbit: technical case report. Neurosurgery 2000;47:778-83.

20. Peker S, Kiliç T, Sengöz M, Pamir MN. Radiosurgical treatment of cavernous sinus cavernous haemangiomas. Acta Neurochir (Wien) 2004;146:337-41; discussion 340.

21. Khan AA, Niranjan A, Kano H, Kondziolka D, Flickinger JC, Lunsford LD. Stereotactic radiosurgery for cavernous sinus or orbital hemangiomas. Neurosurgery 2009;65:914-8; discussion 918.

22. Wang X, Mei G, Liu X, Dai J, Pan L, Wang E. The role of stereotactic radiosurgery in cavernous sinus hemangiomas: a systematic review and meta-analysis. J Neurooncol 2012;107:239-45. 